\title{
"Ordenar la casa": securitización y producción de irregularidad en el norte de Chile
}

\section{Roberto Dufraix Tapia* \\ Romina Ramos Rodríguez** \\ Daniel Quinteros Rojas** (1)}

\section{Resumen}

La zona norte de Chile es considerada como un espacio privilegiado tanto para el intercambio económico como para el tránsito de personas. En efecto, las regiones de Arica y Parinacota, Tarapacá y Antofagasta son las que exhiben las proporciones más altas de población extranjera en todo el país. Este contexto, sin embargo, se contrapone con las políticas de frontera que se han implementado durante la última década y que operan desde una perspectiva que concibe a ciertas movilidades como un auténtico peligro. Esta funcionalidad de la frontera logra dar cuenta de su comportamiento heterogéneo, permisivo para la movilidad de capitales, pero restrictivo para la movilidad humana. En este contexto, el presente trabajo se enfoca en el estudio de dos medidas adoptadas durante el año 2018, que resultan ser particularmente significativas: la imposición de visas consulares para personas provenientes de Haití y Venezuela y el proceso extraordinario de regularización migratoria. Así, a partir de la revisión de los datos oficiales sobre visas y deportaciones, se concluye que la securitización de las migraciones ha construido un régimen de ilegalidad e irregularidad de miles de personas extranjeras en Chile, particularmente en la zona fronteriza del Norte Grande.

Palabras clave: fronteras, migración, control, regularización, producción de irregularidad.

\footnotetext{
* Universidad de Tarapacá, Iquique, Chile.

** Universidad Arturo Prat, Iquique, Chile.
} 


\section{"Put our house in order": securitization and the production of irregularity in the north of Chile}

\section{Abstract}

The northern area of Chile is considered a privileged space both for economic exchange and for the transit of people. Indeed, the regions of Arica and Parinacota, Tarapacá and Antofagasta are those that exhibit the highest proportions of foreign population in the entire country. This context, however, is at odds with the border policies that have been implemented during the last decade and that operate from a perspective that conceives certain mobilities as a real danger. This functionality of the border manages to account for its heterogeneous behavior, permissive for capital mobility, but restrictive for human mobility. In this context, this paper focuses on the study of two measures adopted during 2018 that turn out to be particularly significant for the purposes of this study: the imposition of consular visas for people from Haiti and Venezuela and the extraordinary process of migratory regularization . Thus, from the review of official data on visas and deportations, it is concluded that the securitization of migration has built a regime of illegality and irregularity for thousands of foreigners in Chile, particularly in the border area of the Norte Grande.

Keywords: borders, migration, control, regularization, production of irregularity.

\section{Introducción}

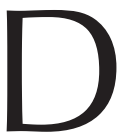

urante las últimas décadas, es posible advertir a nivel global una serie de transformaciones en torno a los espacios fronterizos. En este sentido, entender la frontera como una arquitectura inminentemente heterogénea y móvil (Topak, 2014) permite reconocer su reconfiguración en el marco de los "rebordering" estatales (Walters, 2006) orientados a promover la apertura de las fronteras cuando aquello que se mueve es el capital (Ramos; Tapia, 2019) y a restringir la movilidad de los flujos de migrantes que son considerados como peligrosos (Tarrius, 2000; Adey, 2003). Esta bipolaridad fronteriza (Núñez, 2013) permite el despliegue de una serie de dispositivos de control con el objetivo de contener estas movilidades peligrosas por abajo y facilitar las movilidades 
del capital por arriba (García Pinzón, 2015; Ramos; Ovando, 2016), todo lo cual da cuenta de una estrategia heterogénea de fronteras abiertas y cerradas, que opera según se lea la movilidad (Balibar, 2003).

En el caso de la zona fronteriza del norte de Chile, cuyos límites con Perú y Bolivia se han configurado históricamente sobre la base de un imaginario nacionalista a partir de la Guerra del Pacífico (1879-1883), también es posible observar dinámicas fronterizas de apertura y cierre. Lejos de aquella concepción "dura" de las fronteras, heredada del proceso de "chilenización" durante la postguerra, desde 1975 comienza un proceso orientado hacia su apertura, con la creación de una zona franca en la región de Tarapacá (González, 1992), la cual, para ese entonces, también comprendía el territorio de la actual región de Arica y Parinacota. Este primer gran hito es altamente relevante si se considera que, a partir de ahí, se generaron condiciones privilegiadas no sólo para el intercambio comercial desde el Centro Oeste Sudamericano hacia el mercado del Asia Pacífico (Aranda; Ovando; Corder, 2010), sino también para la movilización sostenida de bienes y capitales dentro de la subregión andina (Tapia, 2012).

Sobre esta base, Chile intensificó el proceso de internacionalización de su economía durante la década del 1990, al firmar un Tratado de Libre Comercio (TLC) con Canadá y, luego, un Acuerdo de Complementación Económica (ACE) con el Mercosur. Como resultado, Chile posee actualmente la mayor red de Tratados de Libre Comercio del mundo (OCDE, 2018), con 29 acuerdos comerciales, que involucran a 65 países, lo cual le permite acceder a un mercado que alcanza al $67 \%$ de la población mundial y al 88\% del PIB mundial (Subsecretaría de Relaciones Internacionales, 2020). En este contexto, los compromisos internacionales en materia comercial obligan al Estado chileno a mantener una política de fronteras abiertas para los flujos del capital, lo cual exige repensar la seguridad de aquellos territorios críticos que ponen a prueba su atractiva estabilidad para la inversión extranjera ${ }^{1}$.

\footnotetext{
${ }^{1}$ Las zonas consideradas críticas son aquellos espacios donde su extensión territorial amplia y escasos controles fronterizos facilitan la movilidad de personas por pasos no habilitados, la trata de personas y tráfico de migrantes, entre otros delitos transnacionales (García Pinzón, 2016).
} 
En consecuencia, junto a las estrategias de apertura comercial, se ha observado también la progresiva consolidación de una estrategia gubernamental de securitización migratoria, fuertemente influenciada por la Doctrina de la Seguridad Nacional (Stang, 2016). De forma llamativa, este proceso también encuentra su hito fundacional en 1975 con la entrada en vigencia de la Ley de Extranjería contenida en el Decreto Ley 1094 (DL1094), la cual ha entregado amplios márgenes de discrecionalidad a los agentes de control migratorio para prohibir el ingreso en frontera, realizar detenciones que han sido luego declaradas ilegales por los tribunales superiores de justicia, o bien, aplicar selectiva y discrecionalmente los mecanismos sancionatorios, particularmente las expulsiones (Quinteros, 2016).

Por una parte, desde el año 2011, esta dinámica de securitización se ha intensificado con la implementación del Plan Frontera Norte 2011-2014, el cual destinó más de USD60 millones para el combate del narcotráfico en las tres regiones del extremo norte del país: Arica y Parinacota, Tarapacá y Antofagasta (García Pinzón, 2016). Dicho programa fue reformulado y expandido durante el año 2018 a las regiones de Atacama y Coquimbo, buscando reforzar el control fronterizo con el uso de tecnología y la coordinación de diversas agencias estatales, creando así las condiciones necesarias para sellar los límites y anticiparse a los riesgos (Ramos; Tapia, 2019). Por otra parte, durante este mismo período, la política migratoria ha restringido fuertemente el acceso a la regularidad, a través de la eliminación de la visa temporal por motivos laborales y la sucesiva incorporación, como requisito para el ingreso, de visas consulares para determinados países. Así, nacionales de República Dominicana, Haití y Venezuela han debido comenzar a obtener sus visas en el país de origen, lo que ha provocado no sólo una baja en los ingresos totales, sino también un aumento del ingreso clandestino (Cerda, 2019).

En suma, el afán de este trabajo es aportar a un área de interés que ha surgido en el último tiempo en los estudios migratorios chilenos, y que se nutre de la discusión respecto al fortalecimiento de las fronteras a partir de nuevos dispositivos de control y vigilancia (García Pinzón, 2015; Liberona, 2015; Quinteros, 2016; Stang, 2016; Ramos; Ovando, 2016; 
Stefoni; Stang, 2017; Thayer; Stang; Abarca, 2016; Aedo, 2017; Tapia; Liberona; Contreras, 2017). En este sentido, se pretende explorar cómo las dinámicas de securitización que se han desarrollado en el norte de Chile afectan la regularidad de la población migrante que habita y transita por este espacio, para lo cual se analizarán dos políticas gubernamentales de gestión migratoria y fronteriza implementadas durante los últimos años, para estudiar luego el efecto que éstas tuvieron sobre el régimen chileno de deportaciones, el cual ha experimentado una significativa transformación durante la última década. En concreto, el estudio se centrará en el impacto que tuvieron tanto la aplicación de visas consulares como el último proceso de regularización migratoria del año 2018, el que surgió como respuesta al aumento de la irregularidad en el contexto de un sostenido aumento de la inmigración durante toda la década. Para ello, se presenta un análisis estadístico descriptivo tanto de las visas otorgadas como de las expulsiones dictadas y ejecutadas por las agencias estatales entre los años 2010 y 2019, las cuales han sido obtenidas a través de diversas solicitudes de acceso a la información pública, gracias a la Ley de Transparencia n. 20.285.

De este modo, el itinerario del presente artículo es el siguiente: en una primera parte se discute, a nivel teórico, el rol de las fronteras en los procesos de securitización de las migraciones. A continuación, se describen las medidas implementadas por el Estado chileno durante la última década para gestionar y controlar el ingreso y permanencia de personas extranjeras. En concreto nos centramos, por una parte, en la política de visas consulares y, por otra, en el último proceso de regularización extraordinario de 2018. Luego, se presentan los resultados de estos procesos, analizando particularmente su impacto en las transformaciones que ha experimentado el sistema de deportaciones chileno. Finalmente, se discute la producción de irregularidad migratoria a partir de las estrategias de apertura y cierre de la frontera que se observan en la zona norte de Chile. 


\section{Securitización de las migraciones y el rol de las fronteras}

Las fronteras cumplen un rol clave en la gestión de la seguridad, porque son vistas como un espacio para todo tipo de actividades ilegales y potencialmente peligrosas (Balibar, 2003; 2005). Los bordes de la nación no son impermeables, pero tampoco se desvanecen, sino que se reformulan para cumplir nuevos propósitos mediante un sofisticado proceso de desborde y rebordeo (Sassen, 2007). Esta reconfiguración permite que actúen como un instrumento discriminatorio encargado de clasificar las poblaciones en movimiento (Walters, 2006; Topak, 2014). En efecto, en lugar de actuar como muros que detienen indiscriminadamente todos los flujos, en un mundo globalizado las fronteras son "inteligentes" y vigilan selectivamente ciertos flujos, mientras permiten que otros avancen sin interrupciones: "operan en una coyuntura crítica entre las expectativas de seguridad y los intensos intercambios comerciales" (Deleixhe; Dembinska; Danero Iglesias, 2019, p. 640). En este contexto de (in)seguridad, las fronteras logran desplazarse hacia otros espacios, como embajadas, aeropuertos, control de identidad preventivos en la vía pública, departamentos de extranjería, por mencionar algunos (Bigo, 2006; 2008; Parker; Vaughan-Williams, 2009; Mezzadra; Neilson, 2013a; 2013b).

La seguridad, desde una perspectiva constructivista (Waever, 1998), se comprende en términos de procesos, como un fenómeno multidimensional que puede involucrar diversos objetos (Baeza, 2016). La seguridad es una definición política, razón por la cual "securitizar" implica que la política, a través de medidas excepcionales, coloca al objeto de referencia en modo de emergencia "ante una amenaza existencial que permite, paradójicamente, sustraer del ámbito de lo político a dicho objeto y así, propiciar su 'tratamiento

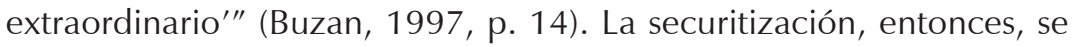
caracteriza por prácticas de excepcionalidad, donde los Estados generan diversos perfiles de riesgo y, para el caso de las migraciones transnacionales, 
una serie de "marcos normativos imprescindibles de la movilidad" (Bigo, 2006, p. 47).

En concreto, la securitización de la migración refiere a la apropiación del término "seguridad nacional" en el gobierno de las migraciones (Domenech, 2013; 2017; Stefoni; Stang, 2017). Es decir, la migración subraya la naturaleza transnacional en la gestión de la seguridad y, como respuesta, se propone su tratamiento securitario (Campesi, 2012) que, para el caso chileno, se enmarca principalmente en el sostenido aumento de los flujos inmigratorios durante las últimas décadas (INE, 2020). En efecto, el sustrato que guía estas narrativas se sustenta en el imaginario del "enemigo interno", teniendo como marco de referencia la legislación migratoria más antigua del continente, la que concibe a la migración como una amenaza para la seguridad del Estado (Stang, 2016). De este modo, es posible, entonces, entender que las medidas de penalización y restricción del ingreso de extranjeros forman parte de una gobernanza migratoria de securitización (Stang; Stefoni, 2016). Lo anterior porque contempla medidas que prohíben el ingreso de ciertos migrantes y otorgan un amplio margen de discrecionalidad a las autoridades, mediante políticas enfocadas en resguardar la seguridad nacional y en que el acceso de las personas migrantes a sus derechos depende del propio estatus de regularidad (Echagüe, 2018; Concha, 2018; Quinteros; Dufraix; Ramos, 2019).

Con todo, se observa que la globalización no ha borrado las fronteras, sino que ha reformulado sus funciones y las ha convertido en instrumentos políticos clave para controlar las actividades transnacionales irregulares y clandestinas (Sassen, 2007). Así, algunos flujos transnacionales se han favorecido, mientras que "otros han sido sometidos a un escrutinio y monitoreo intensivos, lo que lleva a su securitización" (Deleixhe et al., 2019, p. 645). De esta forma, los gobiernos securitarios son especialmente significativos en los territorios fronterizos, donde la movilidad humana se presenta como una amenaza para la seguridad interna, cultural y económica (Huysmans, 2006). 


\section{Regularización y securitización: la respuesta a la irregularidad migratoria en Chile}

El 23 de abril de 2018, a la par del anuncio de una nueva ley de migraciones, el Gobierno chileno comunicó una serie de medidas en materia de extranjería y control de fronteras. Junto a la presentación de este proyecto de ley, que aún se encuentra en discusión parlamentaria, se llevó a cabo un proceso extraordinario de regularización migratoria; se aumentaron las restricciones de ingreso para venezolanos y haitianos mediante la imposición de una visa consular de turismo; se eliminó la visa temporaria por motivos laborales; se fortalecieron los mecanismos para ejecutar expulsiones; y se implementó el Plan Frontera Segura en las cinco regiones del norte chileno. Al momento de socializar este paquete de medidas, el presidente Sebastián Piñera señaló que "llegó la hora de poner orden en este hogar que compartimos", adoptando con ello una narrativa securitaria en la cual conviven discursos contradictorios que buscan promover, por una parte, políticas de frontera abierta para una migración ordenada y regular y, por otra, de frontera cerrada para la migración "ilegal", la trata de personas y el tráfico de migrantes ${ }^{2}$.

Estas medidas buscaban dar respuesta a la situación de irregularidad en la que se encontraban miles de extranjeros y extranjeras en Chile hasta ese año, debido a la concurrencia de, al menos, tres factores. Primero, es necesario considerar el progresivo aumento de la población extranjera en Chile, la que, según las últimas estimaciones del Instituto Nacional de Estadísticas (2019; 2020), pasó de representar el 1,2\% de la población nacional en 2002 al 7,8\% en 2019 (1.492.522). Segundo, el año 2015 se implementó la visa temporaria por motivos laborales, la que permitía regularizar la situación de quienes ingresaban con visa de turismo y cambiaban su calidad migratoria al encontrar trabajo durante su estadía

${ }^{2}$ Medio de prensa nacional Cooperativa, 2018: anuncio de la nueva ley de migraciones con el que el presidente pretende ordenar la casa. Disponible en: https://www.cooperativa./ noticias/pais/poblacion/inmigrantes/ley-de-migracion-el-proyecto-con-que-el-presidentepinera-quiere/2018-04-09/120528.html. 
en el país. Esta medida administrativa permitió aumentar de 54.000 visas temporarias en promedio para el período 2011-2014 a 141.679 en 2015 (Departamento de Extranjería y Migración, 2016; 2017). En la práctica, su eliminación obligó a que buena parte de quienes se encontraban en esta categoría tuvieran que acogerse al proceso de regularización que se implementó como parte del mismo paquete de medidas. Tercero, el año 2012 se establece la obligación de obtener la visa consular para República Dominicana, lo que implicó una drástica reducción en el otorgamiento de visas para sus nacionales y un negativo impacto en el ingreso clandestino.

\section{Visas consulares}

Por regla general, de acuerdo con lo establecido en los artículos 45 y siguientes del DL 1094, el Estado de Chile no exige visas para ingresar al territorio nacional en calidad de turistas. Sin embargo, durante los últimos lustros ha sido posible observar un mayor uso de este tipo de medidas, particularmente respecto de las nacionalidades que constituyen los nuevos flujos migratorios hacia Chile, donde destaca, por ejemplo, el caso de República Dominicana. En efecto, frente al incremento que venía experimentando tal flujo desde la primera década del presente siglo, el Estado de Chile decidió imponer, durante el año 2012, la obligación de visado para todos aquellos nativos y nativas de República Dominicana que pretendieran ingresar a Chile en calidad de turistas. De ahí que, de acuerdo a cifras del Instituto Nacional de Migraciones de República Dominicana (INMRD), la cantidad de visas otorgadas a sus nacionales para entrar a Chile sufriera una importante caída, pasando de 4.390 en 2012 a 2.536 en el 2014 (INMRD, 2018).

Esta restricción de acceso a la regularidad migratoria, sin embargo, lejos de interrumpir el flujo, ha terminado incentivando el ingreso irregular al país, lo que se evidencia cuando se observa el tipo de infracciones por el que han sido sancionados los y las nacionales de dicho país, particularmente desde la entrada en vigencia del respectivo visado. Según la información 
disponible en el Departamento de Extranjería y Migración, el 90\% de las expulsiones decretadas contra dominicanos y dominicanas durante el 2013 fue con motivo de algún ingreso clandestino por paso no habilitado o eludiendo el control policial (Galaz; Rubilar; Silva, 2016).

Pese a lo anterior, el Estado de Chile ha insistido en la generación de visas consulares para regular los flujos. Así ha ocurrido respecto de los casos de Haití y Venezuela. En el caso de Haití, por ejemplo, la necesidad de contar con visas de turismo existe a partir del Decreto n. 776 del 09 de abril del 2018. En particular, este decreto destaca porque, junto con restringir el ingreso al país de nativos y nativas de Haití, justifica tal decisión en la necesidad de protección frente a las redes de tráfico de personas y, en general, a "los riesgos derivados de su situación irregular en el país" (Considerando n. 3 del Decreto n. 776 del Ministerio del Interior y Seguridad Pública).

Lo anterior permite apuntar dos cuestiones que ya han sido advertidas por la literatura. Por una parte, como bien ha sostenido Thayer (2018), la pretensión de reducir el flujo migratorio por la vía de las visas consulares no hace más que precarizarlo e irregularizarlo, pero sin nunca llegar a detenerlos. Por otra, permite advertir el despliegue de una estrategia de control de flujos que, fundada aparentemente en un discurso basado en la protección de la población migrante, tiende a agudizar las restricciones que existen para obtener la regularidad y, de este modo, administrar con un mayor grado de eficacia los flujos (Domenech, 2013).

\section{Regularización migratoria}

Sumado a la imposición de visas consulares para ciertas nacionalidades, el Estado chileno implementó, durante el 2018, un nuevo proceso de regularización. Tras los anteriores procesos de regularización migratoria, llevados a cabo en 1998 y 2007, el Estado chileno dispuso una medida extraordinaria para resolver, en principio, la situación de las casi 300.000 personas que, se estimaba, participarían de la convocatoria, promoviendo 
con ello una migración "ordenada, segura y regular". Sin embargo, según la Subsecretaría del Interior (2018), durante los tres meses en que se mantuvo abierto el proceso, tan sólo 155.707 migrantes presentaron sus solicitudes. Ello motivó diversos cuestionamientos desde la sociedad civil ${ }^{3}$, ya que, a pesar de ser considerado un avance en el reconocimiento y protección de derechos para la población migrante, se llevó a cabo con altos niveles de desinformación, tanto en las instituciones responsables en tramitar las solicitudes, como en la comunidad de extranjeros residentes en Chile. En efecto, un estudio realizado por el Instituto Nacional de Derechos Humanos (2018) en las oficinas preparadas para atender las solicitudes da cuenta de que el $50 \%$ de los extranjeros no conocía los plazos para inscribirse, un $42 \%$ no conocía los requisitos del proceso y el $40 \%$ señaló que en estos lugares no había ningún funcionario público que aclarara sus dudas.

Tabla 1 - Cantidad, porcentaje y tasa (cada 100.000 habitantes) de visas otorgadas en Proceso de Regularización Extraordinaria 2018 en Chile, por región y nacionalidad

\begin{tabular}{|c|c|c|c|c|c|c|c|c|c|}
\hline REGIÓN & $\sum_{0}^{\nwarrow}$ & 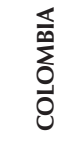 & $\frac{\mathrm{E}}{\mathbf{x}}$ & 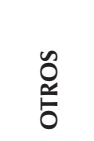 & 总 & $\begin{array}{l}\text { 䍃 } \\
\frac{\mathrm{N}}{\mathrm{W}} \\
\end{array}$ & $\underset{\mathfrak{L}}{\stackrel{\underline{\sigma}}{O}}$ & $\therefore$ & 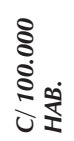 \\
\hline $\begin{array}{l}\text { Arica y } \\
\text { Parinacota }\end{array}$ & 814 & 262 & 6 & 615 & 1.387 & 161 & 3.245 & $2,5 \%$ & 1.473 \\
\hline Tarapacá & 6.962 & 918 & 95 & 2.032 & 2.457 & 408 & 12.872 & $9,8 \%$ & 4.031 \\
\hline Antofagasta & 3.125 & 2.405 & 15 & 506 & 931 & 377 & 7.359 & $5,6 \%$ & 1.288 \\
\hline Metropolitana & 1.618 & 8.200 & 22.090 & 9.472 & 14.974 & 18.542 & 74.896 & $57,0 \%$ & 1.076 \\
\hline $\begin{array}{l}\text { Otras } \\
\text { Regiones }\end{array}$ & 812 & 2.976 & 18.762 & 3.443 & 1.095 & 5.939 & 33.027 & $25,1 \%$ & 364 \\
\hline TOTAL & 13.331 & 14.761 & 40.968 & 16.068 & 20.844 & 25.427 & 131.399 & $100 \%$ & 767 \\
\hline
\end{tabular}

Fuente: elaboración propia en base a datos de la Subsecretaría del Interior (2020a).

${ }^{3}$ Medio de prensa nacional La Pausa (2019) "Situación de migrantes en Chile llega a la Comisión Interamericana de Derechos Humanos". Disponible en: https:/www.pauta.cl/ cronica/situacion-de-migrantes-chile-llega-a-la-comision-interamericana-ddhh. 
En la etapa siguiente se inició un proceso de revisión y análisis de la información obtenida en los registros, tras lo cual se procedió a la entrega de visados para quienes habían sido admitidos. A partir de lo informado por el Gobierno chileno, la Tabla 1 muestra las visas otorgadas como resultado del Proceso de Regularización Extraordinaria 2018, según nacionalidad y región. Si bien destaca la gran cantidad y proporción de visas entregadas en la región Metropolitana, al controlar por el tamaño poblacional informado para cada región en el último Censo de 2017, queda en evidencia el peso relativo que tiene este fenómeno en las regiones del Norte Grande. En este sentido, por ejemplo, Tarapacá cuadruplica la tasa de visas otorgadas a cada 100.000 habitantes en la región Metropolitana, llegando incluso a decuplicar lo observado en algunas regiones del sur del país. Por otra parte, al revisar la regularización por nacionalidad, es posible advertir que una de cada dos personas era nacional de Haití $(31,2 \%)$ o Venezuela $(19,4 \%)$ y, en menor medida, de Perú (15,9\%), Colombia $(11,2 \%)$ o Bolivia $(10,1 \%)$.

En contraste, el resultado de la operación también muestra que una de cada seis solicitudes fue rechazada por registrar antecedentes penales en sus comunidades de origen o haber ingresado sus solicitudes después del periodo habilitado (Briones, 2019). A ello es posible sumar otras 43 mil solicitudes que, no obstante haber sido aprobadas, aún no lograban estampar el permiso de residencia en su pasaporte hacia el término del proceso ${ }^{4}$. En este último caso, el problema se concentró en la obtención del certificado de antecedentes penales apostillados por los organismos oficiales en las comunidades de origen, requisito obligatorio para materializar la regularización. Por su parte, dentro de los motivos que dificultaron la obtención de este documento, se identifican los altos costos para tramitar el certificado en las comunidades de origen y/o la lentitud de las embajadas en procesar las solicitudes. En ambos casos, la autoridad ha señalado que lo que único que queda por hacer es abandonar el territorio o exponerse a la expulsión administrativa por irregularidad migratoria. En esta línea,

${ }^{4}$ Medio de prensa nacional CNN Chile (2019) "uno de cada 6 inmigrantes no superó el Proceso de Regularización del gobierno: Podrían ser expulsados". Disponible en: https:// www.cnnchile.com/pais/1-de-6-inmigrantes-no-supero-expulsion_20191002/. 
el vocero de la Coordinadora Nacional de Inmigrantes, Rodolfo Noriega, aseguró que la tardanza en la tramitación de cada caso ha potenciado la irregularidad de migrantes (Briones, 2019), cuestión que finalmente fue, como se verá, uno de los principales resultados de esta política.

\section{La expulsión administrativa como mecanismo de producción de irregularidad}

Las medidas de regularización migratoria que se han discutido hasta acá se desarrollaron en el marco de una notable expansión, diversificación y transformación del sistema de deportaciones chileno. Desde el año 2012, a la expulsión por motivos administrativos que regía en Chile desde el año 1918 y que se modifica con el DL1094 de 1975, se agrega la expulsión como una pena alternativa a la privación de libertad por la comisión de un delito. Ello ha permitido, por una parte, que las expulsiones efectivamente ejecutadas se hayan triplicado entre 2010 y 2019 (ver Tabla 2), pasando de 763 a 2232, con una progresiva importancia de las deportaciones penales (Brandariz; Dufraix; Quinteros, 2018). Sin embargo, por otra parte, esta creciente priorización de las expulsiones penales ha significado una baja considerable en la materialización de expulsiones administrativas, las que pasaron de representar casi un 90\% el año 2013 (1068) a tan sólo un 25\% en 2019 (576). Lo anterior implica, por tanto, un aumento considerable en la brecha que se produce entre las expulsiones administrativas que son dictadas respecto de las que son ejecutadas, lo que ha sido apuntado en diferentes países por la literatura internacional bajo la idea de (Brandariz, en prensa).

Por ello, dentro del paquete de medidas anunciado por el gobierno chileno en 2018, se consideraba el fortalecimiento del sistema de deportaciones para agilizar y mejorar los procedimientos de expulsión. Lo anterior buscaba dar respuesta, en parte, al aumento del stock de extranjeros y extranjeras sobre las cuales pesaban órdenes administrativas de expulsión sin materializar (Inostroza, 2019). En este sentido, a partir de 
la información que se presenta en el Gráfico 1, es posible desprender que, entre 2010 y 2017, un 60,5\% de las expulsiones administrativas no lograron concretarse, acumulando con ello un total de 11.403 órdenes sin ejecutar durante este período. Más aún, esta situación contrasta notoriamente con las expulsiones penales, donde la efectividad está prácticamente garantizada, particularmente debido a que más del 99\% de este grupo de personas se encontraba en prisión preventiva al momento de su expulsión (Defensoría Penal Pública, 2020).

Tabla 2 - Cantidad de expulsiones administrativas y penales ejecutadas en Chile, por región y año

\begin{tabular}{l|c|c|c|c|c|c|c|c|c|c}
\multicolumn{1}{c|}{ REGIÓN } & $\mathbf{2 0 1 0}$ & $\mathbf{2 0 1 1}$ & $\mathbf{2 0 1 2}$ & $\mathbf{2 0 1 3}$ & $\mathbf{2 0 1 4}$ & $\mathbf{2 0 1 5}$ & $\mathbf{2 0 1 6}$ & $\mathbf{2 0 1 7}$ & $\mathbf{2 0 1 8}$ & $\mathbf{2 0 1 9}$ \\
\hline Arica y Parinacota & 288 & 345 & 316 & 191 & 163 & 156 & 125 & 225 & 239 & 170 \\
\hline Tarapacá & 142 & 197 & 174 & 418 & 499 & 545 & 432 & 730 & 877 & 883 \\
\hline Antofagasta & 169 & 201 & 207 & 138 & 260 & 234 & 157 & 136 & 193 & 192 \\
\hline Metropolitana & 116 & 197 & 185 & 288 & 280 & 227 & 104 & 196 & 584 & 748 \\
\hline Otras Regiones & 48 & 102 & 139 & 140 & 153 & 179 & 99 & 111 & 159 & 239 \\
\hline TOTAL & $\mathbf{7 6 3}$ & $\mathbf{1 . 0 4 2}$ & $\mathbf{1 . 0 2 1}$ & $\mathbf{1 . 1 7 5}$ & $\mathbf{1 . 3 5 5}$ & $\mathbf{1 . 3 4 1}$ & $\mathbf{9 1 7}$ & $\mathbf{1 . 3 9 8}$ & $\mathbf{2 . 0 5 2}$ & $\mathbf{2 . 2 3 2}$
\end{tabular}

Fuente: elaboración propia en base a datos de la Policía de Investigaciones (2019; 2020).

Sin embargo, como puede observarse en el Gráfico 1, lejos de aumentar el nivel de efectividad, las medidas adoptadas por el gobierno chileno durante el año 2018 redujeron drásticamente la proporción de órdenes ejecutadas. Si entre los años 2010 y 2017, sólo un tercio de las expulsiones administrativas lograba materializarse, esa proporción disminuyó a 9,1\% durante el 2018 y 8,6\% para el 2019. En esta dramática baja en el porcentaje de ejecución, si bien influye la menor cantidad de expulsiones administrativas efectivamente realizadas, lo realmente decisivo resulta ser el explosivo aumento en la cantidad de órdenes de expulsión decretadas. Como se observa en la gráfica, entre los años 2010 y 2016, la cantidad de 
decretos difícilmente superaba los 2.500 por año, barrera que se rompe el año 2017, Ilegando incluso a alcanzar las casi siete mil expulsiones el 2019.

\section{Gráfico 1 - Cantidad de expulsiones administrativas decretadas, ejecutadas y} porcentaje de ejecución por año (2010-2019)

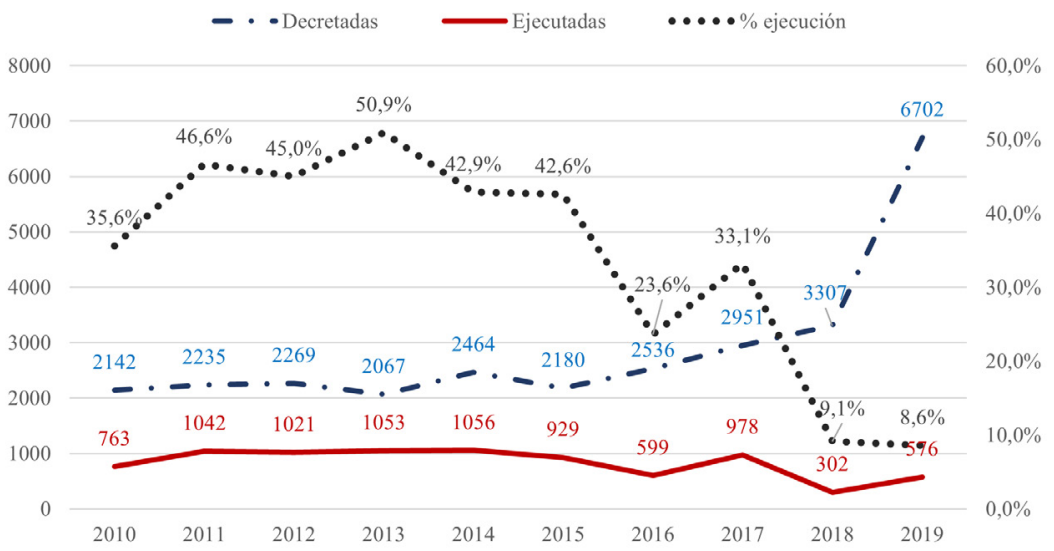

Fuente: elaboración propia en base a datos de la Policía de Investigaciones $(2019 ;$; 2020) y de la Subsecretaría del Interior (2020b; 2020c).

Esta situación se ve particularmente acentuada en el espacio fronterizo del Norte Grande chileno. Entre los años 2010 y 2019, las regiones de Arica y Parinacota, Tarapacá y Antofagasta concentraron el 78\% de los decretos administrativos de expulsión y el $67,7 \%$ del total de expulsiones ejecutadas por la Policía de Investigaciones (2019; 2020). En efecto, al observar los datos de la Tabla 2 es posible observar que, en términos de ejecución, Tarapacá concentró el 36,8\% de las expulsiones durante esta década, las que se materializan principalmente por el paso fronterizo Colchane-Pisiga, que une a las regiones de Tarapacá y Oruro. Sin embargo, la Tabla 3 muestra un notable giro respecto de lo ocurrido con las órdenes de deportación administrativa: si bien hasta el año 2018 la mayor cantidad 
de los decretos eran impuestos por la autoridad administrativa de Tarapacá, para el año 2019 éstos registraron un aumento del 375\% en la región de Arica y Parinacota, la cual llegó a representar el 46,6\% del total de decretos administrativos de expulsión en el país.

Tabla 3 - Cantidad de expulsiones administrativas decretadas en Chile, por año y región

\begin{tabular}{l|l|l|l|l|l|l|l|l|l|l}
\multicolumn{1}{c|}{ REGIÓN } & $\mathbf{2 0 1 0}$ & $\mathbf{2 0 1 1}$ & $\mathbf{2 0 1 2}$ & $\mathbf{2 0 1 3}$ & $\mathbf{2 0 1 4}$ & $\mathbf{2 0 1 5}$ & $\mathbf{2 0 1 6}$ & $\mathbf{2 0 1 7}$ & $\mathbf{2 0 1 8}$ & $\mathbf{2 0 1 9}$ \\
\hline $\begin{array}{l}\text { Arica y } \\
\text { Parinacota }\end{array}$ & 313 & 195 & 185 & 162 & 283 & 271 & 351 & 336 & 658 & 3.126 \\
\hline Tarapacá & 620 & 796 & 999 & 1.014 & 967 & 819 & 858 & 1.143 & 1.224 & 1.817 \\
\hline Antofagasta & 439 & 328 & 373 & 282 & 368 & 245 & 313 & 308 & 185 & 282 \\
\hline Metropolitana & 581 & 657 & 473 & 343 & 537 & 582 & 746 & 831 & 825 & 875 \\
\hline Otras Regiones & 189 & 259 & 239 & 266 & 309 & 263 & 268 & 333 & 415 & 602 \\
\hline TOTAL & $\mathbf{2 . 1 4 2}$ & $\mathbf{2 . 2 3 5}$ & $\mathbf{2 . 2 6 9}$ & $\mathbf{2 . 0 6 7}$ & $\mathbf{2 . 4 6 4}$ & $\mathbf{2 . 1 8 0}$ & $\mathbf{2 . 5 3 6}$ & $\mathbf{2 . 9 5 1}$ & $\mathbf{3 . 3 0 7}$ & $\mathbf{6 . 7 0 2}$
\end{tabular}

Fuente: elaboración propia en base a datos de la Subsecretaría del Interior (2020b; 2020c).

Más aún, al observar la cantidad de expulsiones administrativas decretadas por nacionalidad, es posible identificar algunos acentos territoriales específicos de la gestión migratoria chilena. De acuerdo con los datos que se presentan en la Tabla 4, hasta el año 2018, el 88,2\% de las expulsiones administrativas decretadas se concentraban en nacionales de tan sólo cuatro países: Bolivia (37\%), Colombia (21,6\%), Perú (15,8\%) y República Dominicana (13,8\%). Sin embargo, como resultado del proceso de regularización y de la imposición de visas consulares llevado a cabo durante el año 2018, las órdenes administrativas de deportación se duplicaron para el 2019. Más aún, esto ocurre principalmente respecto de las y los ciudadanos venezolanos, quienes saltaron de 31 a más de dos mil decretos de expulsión en sólo un año. Con ello, las 1.608 órdenes de deportación 
administrativa que se impusieron contra nacionales de Venezuela en la región de Arica y Parinacota durante el año 2019 representaron casi un cuarto del total de expulsiones administrativas decretadas en Chile para ese año (24\%), seguido de las 1.158 órdenes impuestas contra nacionales de Bolivia en Tarapacá (17,3\%).

Tabla 4 - Cantidad de expulsiones administrativas decretadas en Chile, por año y nacionalidad

\begin{tabular}{|c|c|c|c|c|c|c|c|c|c|c|}
\hline Nacionalidad & 2010 & 2011 & 2012 & 2013 & 2014 & 2015 & 2016 & 2017 & 2018 & 2019 \\
\hline Bolivia & 799 & 814 & 942 & 806 & 814 & 824 & 923 & 1.204 & 1.076 & 1.380 \\
\hline Colombia & 219 & 409 & 560 & 694 & 948 & 527 & 465 & 487 & 468 & 651 \\
\hline Perú & 870 & 641 & 446 & 322 & 238 & 280 & 232 & 236 & 238 & 335 \\
\hline $\begin{array}{l}\text { Rep. } \\
\text { Dominicana }\end{array}$ & 12 & 31 & 27 & 73 & 247 & 403 & 724 & 894 & 637 & 885 \\
\hline Cuba & 13 & 28 & 26 & 2 & 5 & 8 & 20 & 24 & 730 & 1.178 \\
\hline Argentina & 108 & 132 & 115 & 73 & 70 & 54 & 51 & 34 & 22 & 20 \\
\hline Ecuador & 46 & 53 & 61 & 45 & 65 & 40 & 37 & 27 & 44 & 52 \\
\hline Paraguay & 11 & 33 & 12 & 5 & 26 & 18 & 10 & 4 & 7 & 0 \\
\hline Brasil & 7 & 16 & 22 & 11 & 11 & 7 & 25 & 4 & 7 & 5 \\
\hline Venezuela & 0 & 5 & 1 & 5 & 7 & 7 & 7 & 5 & 31 & 2.040 \\
\hline Haití & 0 & 6 & 5 & 2 & 6 & 1 & 17 & 6 & 13 & 122 \\
\hline Uruguay & 9 & 11 & 5 & 0 & 3 & 1 & 7 & 5 & 5 & 0 \\
\hline Otros Países & 48 & 56 & 47 & 29 & 24 & 10 & 18 & 21 & 29 & 34 \\
\hline Total & 2.142 & 2.235 & 2.269 & 2.067 & 2.464 & 2.180 & 2.536 & 2.951 & 3.307 & 6.702 \\
\hline
\end{tabular}

Fuente: elaboración propia en base a datos de la Subsecretaría del Interior (2020b; 2020c).

En suma, los datos revelan que la estrategia migratoria y fronteriza adoptada por la administración Piñera durante el año 2018, lejos de contener 
la irregularidad, la profundizó. En primer lugar, es posible observar que la aplicación de visas consulares tuvo un efecto negativo al aumentar significativamente las expulsiones administrativas que se decretaron contra nacionales de República Dominicana desde el año 2014, pero particularmente contra venezolanos y venezolanas durante el año 2019. En segundo lugar, si bien es posible advertir que el proceso de regularización extraordinaria logró la regularización de más de 130 mil personas, no es posible dejar de lado que, a su vez, este proceso terminó en el rechazo de visas para otras tantas miles de personas, quienes irremediablemente deberán ser expulsadas. En tercer lugar, es necesario resaltar el hecho de que todo el proceso administrativo de otorgamiento de visas e imposición de expulsiones se enmarca en el escenario de una considerable disminución en las órdenes administrativas que se ejecutan año a año. En efecto, de las poco más de 11 mil órdenes administrativas de expulsión sin ejecutar entre los años 2010 y 2017, se llegó a las 20.534 en 2019. Con todo, la narrativa de una migración ordenada, segura y regular se hace insostenible frente al aumento de la irregularidad que la misma política produce, la cual termina por profundizar la condición de vulnerabilidad de miles de personas que no podrán acceder a la protección del Estado, por ejemplo, para acceder al mercado regular del trabajo o la vivienda.

\section{Conclusiones}

Reconocemos que la irregularidad no es la única consecuencia que surge de la implementación de políticas migratorias restrictivas. Como bien han planteado Thayer et al., (2016), el estatus legal precario es heterogéneo en términos de categorías migratorias y oscila entre la permanente temporalidad y la plena irregularidad. Sin embargo, a efectos de este estudio, nos centramos en analizar cómo es producida la irregularidad migratoria en Chile a partir de la idea de "ordenar la casa".

En particular, esto ha ocurrido mediante la implementación de dos políticas: la imposición de visas consulares y el proceso de regularización 
migratoria. Estas dos medidas, junto con reforzar la retórica nacionalista que gira en torno a la idea de "ordenar la casa", abre una paradoja en términos de lo que se propone y lo que se obtiene (Concha Villanueva, 2018).

Y esto es exactamente lo que ha sucedido en Chile, a propósito de la gestión de los flujos que considera "no deseados". En este sentido, los datos analizados permiten advertir una profundización de los efectos que trae consigo la irregularidad producida a partir de las políticas analizadas. Así, por ejemplo, la aplicación de visas consulares sobre nacionales de República Dominicana y Venezuela, lejos de contribuir al propósito de "ordenar" tales flujos, generó un aumento significativo de las expulsiones administrativas dictadas en su contra, precarizando aún más su -ya precarizado- estatus de "no ciudadano". Del mismo modo, el proceso de regularización extraordinaria, lejos de cumplir con su propósito declarado, implicó que miles de personas extranjeras quedaran en situación de ser expulsadas.

Así combinadas, estas dos políticas han producido un efecto significativo en el aumento de expulsiones administrativas sin ejecutar. De hecho, las expulsiones administrativas decretadas (y no ejecutadas), con especial énfasis en la zona norte de Chile, abren un escenario complejo en el marco de los derechos de los/as trabajadores/as migrantes, quienes se encuentran obligados/as a aceptar condiciones de trabajo profundamente precarizadas debido a la siempre latente amenaza de expulsión (De Giorgi, 2005; 2012).

Así, los efectos que trae consigo la condición de irregularidad producida por la imposición de políticas migratorias restrictivas, no se reducen únicamente a la expulsión de los flujos "no deseados", sino que además se expanden hacia la disciplina del trabajo precarizado que se deriva precisamente de su condición de sujeto "expulsable" (Aquino, 2015). Como bien han precisado Stefoni, Leiva y Bonhomme (2017, p. 110), la irregularidad migratoria constituye "un factor decisivo a la hora de ofrecer condiciones laborales que violan todas las normas nacionales e internacionales". De este modo, la frontera, y los dispositivos de control que se despliegan a partir de ella, tienden a incapacitar jurídicamente el cuerpo de las personas migrantes, convirtiéndolas en sujetos útiles y funcionales a 
las dinámicas del mercado de trabajo no regulado y, con ello, en una fuerza de trabajo especialmente vulnerable a prácticas explotadoras (De Genova; Peutz, 2010; De Genova, 2015). En consecuencia, la implementación de políticas restrictivas de la movilidad humana no sólo produce la irregularidad; también constituye un terreno fértil para la criminalización de ciertos flujos (Melossi, 2015) y para la proliferación -y normalización- de la explotación del trabajo migrante (De Genova, 2004; De Giorgi, 2012).

Roberto A. Dufraix Tapia es doctor en Sociedad Democrática, Estado y Derecho por la Universidad del País Vasco, Espanha, y docente en la Universidad de Tarapacá, Iquique, Chile.

$\bowtie$ robertodufraix@gmail.com

Romina Ramos Rodríguez es doctora en Sociología y docente e investigadora en la Facultad de Ciencias Jurídicas y Políticas de la Universidad Arturo Prat, Iquique, Chile.

$\triangle$ romramos@unap.cl

Daniel Quinteros Rojas es máster en Criminología por la Universidad de Manchester, Reino Unido, y docente e investigador en la Facultad de Ciencias Jurídicas y Políticas de la Universidad Arturo Prat, Iquique, Chile.

(Autor correspondiente) $₫$ daquintero@unap.cl

\section{Referencias}

1. AEDO, Ángel. Encarnando (in)seguridad. Orden policial y política de la presencia en la frontera norte de Chile. Antípoda - Revista de Antropología y Arqueología, n. 29, p. 87-103, 2017.

2. ADEY, Peter. Secured and sorted mobilities: examples from the airport. Surveillance \& Society, v. 1, n. 4, p. 500-519, 2003.

3. AQUINO, Alejandra. "Porque, si llamas al miedo, el miedo te friega": la ilegalización de los trabajadores migrantes y sus efectos en las subjetividades. Estudios Fronterizos, v. 16, n. 32, p. 75-98, 2015. 
4. ARANDA, Gilberto; OVANDO, Cristian; CORDER, Alejandro. Experiencias paradiplomáticas en la Región de Tarapacá y su proyección subregional. Estudios Internacionales, v. 42, n. 165, p. 33-73, 2010.

5. BAEZA, Matías M. Securitización de las fronteras en América del Sur (20042014). Tesis (Maestría en Estudios Internacionales) - Universidad Torcuato Di Tella, Buenos Aires, 2016.

6. BALIBAR, Étienne. Nosotros, iciudadanos de Europa? Madrid: Editorial Tecnos, 2003.

7. BALIBAR, Étienne. Violencia, identidad y civilidad. Para una cultura política global. Barcelona: Editorial Gedisa, 2005.

8. BIGO, Didier. Security, exception, ban and surveillance. En: LYON, David. Theorizing surveillance. Cullompton, UK: Willan Publishing, 2006. p. 46-68.

9. BIGO, Didier. Globalized (in)security: the field and the ban-opticon. En: BIGO, Didier; TSOUKALA, Anastassia (eds.). Terror, insecurity and liberty: illiberal practices of liberal regimes after 9/11. Londres: Routledge, 2008. p. 10-48.

10. BRANDARIZ, José Á. Crimigración y deportation gap, en una perspectiva global. En: DUFRAIX, Roberto; RAMOS, Romina; QUINTEROS, Daniel. Securitización de las fronteras y criminalización de las migraciones. Santiago: Ediciones Jurídicas de Santiago (EJS) (En prensa).

11. BRANDARIZ, José Á.; DUFRAIX, Roberto; QUINTEROS, Daniel. La expulsión judicial en el sistema penal chileno: ¿hacia un modelo de crimmigration? Política Criminal, v. 13, n. 26, p. 739-770, 2018.

12. BRIONES, Nicolás. Un 15\% de los migrantes no superó proceso de regularización y cifra podría aumentar. Bio Bio Chile, 02 de octubre de 2019. Disponible en: https://www.biobiochile.cl/noticias/nacional/chile/2019/10/02/ un-15-de-los-migrantes-no-supero-proceso-de-regularizacion-la-cifra-podriaaumentar.shtml

13. BUZAN, Barry. Rethinking security after the Cold War. Cooperation and Conflict, v. 32, n. 1, p. 5-28, 1997.

14. CAMPESI, Giuseppe. Migraciones, seguridad y confines en la teoría social contemporánea. Revista Crítica Penal y Poder, n. 3, p. 1-20, 2012.

15. CERDA, Claudio. Denuncia por ingresos clandestino llegan a récord y alcanzan más de 4 mil en junio. El Mercurio, 25 de julio de 2019. Disponible en: https:// digital.elmercurio.com/2019/07/25/C/OP3KRQNN\#zoom=page-width

16. CONCHA VILLANUEVA, Sebástian. Propuestas para regular las migraciones en Chile y la obstinación del securitismo. URVIO Revista Latinoamericana de Estudios de Seguridad, n. 23, p. 110-126, 2018.

17. DEFENSORÍA PENAL PÚBLICA. Respuesta a solicitud de acceso a la información pública no AK005T0000581. 2020. 
18. DE GENOVA, Nicholas. The legal production of Mexican/migrant "illegality". Latino Studies, n. 2, p. 160-185, 2004.

19. DE GENOVA, Nicholas. Extremities and regularities. Regulatory regimes and the spectacle of immigration enforcement. En: JANSEN, Yolande; CELIKATES, Robin; BLOOIS, Joost de (eds.). The irregularization of migration in contemporary Europe. Detention, deportation, drowning. Maryland, US: Rowman \& Littlefield International, 2015. p. 3-14.

20. DE GENOVA, Nicholas; PEUTZ, Nathalie (eds.). The deportation regime: sovereignty, space, and the freedom of movement. Durham: Duke University Press, 2010.

21. DE GIORGI, Alessandro. Tolerancia cero. Estrategias y prácticas de la Sociedad de control. Barcelona: Editorial Virus, 2005.

22. DE GIORGI, Alessandro. Control de la inmigración, post- fordismo y menor elegibilidad: una crítica materialista de la criminalización de la inmigración en Europa. Revista Crítica Penal y Poder, n. 2, p. 139-162, 2012

23. DELEIXHE, Martin; DEMBINSKA, Magdalena; IGLESIAS, Julien. Securitized Borderlands. Journal of Borderlands Studies, v. 34, n. 5, p. 639-647, 2019.

24. DEPARTAMENTO DE EXTRANJERÍA Y MIGRACIÓN. Migración en Chile 2005-2014, Santiago: DEM, 2016. Disponible en: https://www.extranjeria.gob.cl/ media/2019/04/Anuario.pdf

25. DEPARTAMENTO DE EXTRANJERÍA Y MIGRACIÓN. Estadísticas migratorias del Departamento de Extranjería y Migración 2015. Santiago: DEM, 2017. Disponible en: https://www.extranjeria.gob.cl/media/2019/04/ AnuarioEstadisticoNacionalDEM2015.pdf

26. DOMENECH, Eduardo. Las migraciones son como el agua: hacia la instauración de políticas de "control con rostro humano". Polis, v. 12, n. 35, p.119-142, 2013.

27. DOMENECH, Eduardo. Las políticas de migración en Sudamérica: elementos para el análisis crítico del control migratorio y fronterizo. Terceiro Milênio Revista Crítica de Sociologia e Política, v. 8, n. 1, p. 19-48, 2017.

28. ECHAGÜE, Clive. "Incivilidades". Notas sobre cómo la intervención estatal nocturna en el centro de Antofagasta endurece "las fronteras". Polis, v. 17, n. 51, p. 39-61, 2018.

29. GALAZ, Caterine; RUBILAR, Gabriela; SILVA, Claudia. Migración dominicana en Chile. Boletín Informativo n. 2. Departamento de Extranjería y Migración. Santiago: DEM, 2016.

30. GARCÍA PINZÓN, Viviana. Estado y frontera en el norte de Chile. Revista de Estudios Fronterizos, v. 16, n. 31, p. 117-148, 2015.

31. GARCÍA PINZÓN, Viviana. Territorios fronterizos: agenda de seguridad y narcotráfico en Chile: el Plan Frontera Norte. Estudios Internacionales, v. 47, n. 181, p. 69-93, 2016. 
32. GONZÁLEZ, Sergio. La zona franca de lquique y su perspectiva industrial: un dilema regional. Revista EURE, v. 18, n. 54, p. 79-90, 1992.

33. HUYSMANS, Jef. The politics of insecurity. Fear, migration and asylum in the EU. Londres: Routledge, 2006.

34. INOSTROZA, Daniel. Hay 12 mil personas que no cumplen los requisitos y ahora el desafío es la expulsión. Economía y Negocios, 02 feb. 2019. Disponible en: http://www.economiaynegocios.cl/noticias/noticias.asp?id=543474

35. INSTITUTO NACIONAL DE DERECHOS HUMANOS. Resultados de observación del proceso de regularización. INDH, 28 abr. 2018. Disponible en: https://www.indh.cl/indh-entrega-resultados-de-observacion-de-proceso-deregularizacion-migratoria-y-valora-la-iniciativa/

36. INSTITUTO NACIONAL DE ESTADÍSTICAS. Estimaciones y proyecciones de la población de Chile 2002-2035. Totales regionales, población urbana y rural. Síntesis de Resultados. Santiago: INE, 2019. Disponible en: https:// www.ine.cl/docs/default-source/proyecciones-de-poblacion/publicaciones-yanuarios/base-2017/ine_estimaciones-y-proyecciones-2002-2035_base-2017 reg_\%C3\%A1 rea_s\%C3\%ADntesis.pdf?sfvrsn=aaeb88e7_5

37. INSTITUTO NACIONAL DE ESTADÍSTICAS. Estimación de personas extranjeras residentes habituales en Chile al 31 de diciembre 2019. Santiago: INE, 2020. Disponible en: https://www.ine.cl/docs/default-source/ demografia-y-migracion/publicaciones-y-anuarios/migraci\%C3\%B $3 n$ internacional/estimaci \% C 3\%B 3n-poblaci\%C 3\%B 3n-extranjera-enchile-2018/estimaci\%C3\%B3n-poblaci\%C3\%B3n-extranjera-en-chile-2019metodolog\%C3\%ADa.pdf?sfvrsn=5b145256_6

38. INSTITUTO NACIONAL DE MIGRACIÓN DE LA REPÚBLICA DOMINICANA. Gobernanza migratoria y políticas públicas: un análisis descriptivo sobre la migración dominicana hacia chile en el período 2005-2018. Santo Domingo: Ministerio de Interior y Policía, 2018. Disponible en: http://inm.gob.do/ transparencia/phocadownload/Publicaciones/INFORME\%20EMIGRACIN\%20 DOMINICANA\%20A\%20CHILE\%2029112918.pdf

39. LIBERONA, Nanette. La frontera cedazo y el desierto como aliado. Prácticas institucionales racistas en el ingreso a Chile. Polis, v. 14, n. 42, p. 143-165, 2015.

40. MELOSSI, Dario. Crime, punishment and migration. London: Editorial SAGE, 2015.

41. MEZZADRA, Sandro; NEILSON, Brett. Between inclusion and exclusion: on the topology of global space and borders. Theory, Culture \& Society, v. 29, n. 4-5, p. 58-75, 2013 a.

42. MEZZADRA, Sandro; NEILSON, Brett. Border as method, or, the multiplication of labor. Durham: Duke University Press, $2013 \mathrm{~b}$.

43. NúÑEZ, Andrés. La frontera no deja ver la montaña: invisibilización de la cordillera de Los Andes en la Norpatagonia chileno-argentina. Revista de Geografía Norte Grande, n. 55, p. 89-108, 2013. 
44. ORGANIZACIÓN PARA LA COOPERACIÓN Y DESARROLLO. Economic surveys 2018: Chile. Paris: OECD, 2018. Disponible en: http://www.oecd.org/ economy/panorama-economico-chile/

45. PARKER, Noel et al. Lines in the sand? Towards an agenda for critical border studies. Geopolitics, v. 14, n. 3, p. 582-587, 2009.

46. POLICÍA DE INVESTIGACIONES. Respuesta a solicitud de acceso a la información pública no AD010T0006483, 2019.

47. POLICÍA DE INVESTIGACIONES. Respuesta a solicitud de acceso a la información pública no AD010T0009112, 2020.

48. QUINTEROS, Daniel. ¿Nueva "crimigración" o la vieja economía política del castigo? Dos aproximaciones criminológicas para entender el control punitivo de la migración en Chile. Astrolabio, n. 17, p. 81-113, 2016.

49. QUINTEROS, Daniel; DUFRAIX, Roberto; RAMOS, Romina. Human trafficking cases in Chile: challenges for reducing the "dark figure". En: WINTERDYK, John; JONES, Jackie (eds.). The Palgrave international handbook of human trafficking, Londres, New York, Xangai: Palgrave Macmillan, 2019. p. 1151-1164.

50. RAMOS, Romina; OVANDO, Cristian. La región de Tarapacá: seguridad fronteriza y múltiples apropiaciones de su espacio. Polis, v. 15, n. 44, p. 57-81, 2016.

51. RAMOS, Romina; TAPIA, Marcela. Una mirada heterogénea del espacio fronterizo: el caso de la frontera tarapaqueña (Chile). Revista CIDOB d'Afers Internacionals, n. 122, p. 187-210, 2019.

52. SASSEN, Saskia. Una sociología de la globalización. Buenos Aires: Katz Editores, 2007.

53. STANG, María F. De la doctrina de la seguridad nacional a la gobernabilidad migratoria: la idea de seguridad en la normativa migratoria chilena, 1975-2014. Polis, v. 15, n. 44, p. 83-107, 2016.

54. STANG, María F.; STEFONI, Carolina. La microfísica de las fronteras. Criminalización, racialización y expulsabilidad de los migrantes colombianos en Antofagasta, Chile. Astrolabio, n. 17, p. 42-80, 2016.

55. STEFONI, Carolina; STANG, María F. La construcción del campo de estudio de las migraciones en Chile: notas de un ejercicio reflexivo y autocrítico. Íconos - Revista de Ciencias Sociales, n. 58, p. 109-129, 2017.

56. STEFONI, Carolina; LEIVA, Sandra; BONHOMME, Macarena. Migración internacional y precariedad laboral: el caso de la industria de la construcción en Chile. Remhu - Rev Interdiscip Mobil Hum Brasília, v. 25, n. 49, p. 95-112, 2017.

57. SUBSECRETARIA DE RELACIONES INTERNACIONALES. Acuerdo comerciales de Chile, 2019. Disponible en: https://www.subrei.gob.cl/modulode-acuerdos-comerciales/ 
58. SUBSECRETARÍA DEL INTERIOR. Presidente Piñera cuenta del proceso de regularización en Chile. Departamento de prensa, 2018. Disponible en: https:// www.subinterior.gob.cl/sin-categoria/2018/07/25/presidente-pinera-inauguronueva-oficina-de-extranjeria-y-migracion-y-dio-a-conocer-balance-del-procesode-regularizacion/

59. SUBSECRETARÍA DEL INTERIOR. Respuesta a Solicitud de Acceso a la Información Pública n. AB001T0001365, 2020 a.

60. SUBSECRETARÍA DEL INTERIOR. Respuesta a Solicitud de Acceso a la Información Pública n. AB001C0004251, 2020b.

61. SUBSECRETARÍA DEL INTERIOR. Respuesta a Solicitud de Acceso a la Información Pública n. AB001T0001403, 2020c.

62. TAPIA, Marcela. Frontera y migración en el norte a partir del análisis de los censos población: siglos XIX- XXI. Revista de Geografía Norte Grande, n. 53, p. 177-198, 2012.

63. TAPIA, Marcela; LIBERONA, Nanette; CONTRERAS, Yasna. El surgimiento de un territorio circulatorio en la frontera chileno-peruana: estudio de las prácticas socio-espaciales fronterizas. Revista de Geografía Norte Grande, n. 66, p. 117141, 2017.

64. TARRIUS, Alain. Leer, describir, interpretar las circulaciones migratorias: conveniencia de la noción de 'territorio circulatorio'. Los nuevos hábitos de la identidad. Relaciones, v. 21, n. 83, p. 37-66, 2000.

65. THAYER, Luis E.; STANG, Maria F.; ABARCA, Cristóbal. Estatus legal precario y condicionalidad en el acceso a derechos: una aproximación a la regulación migratoria de Argentina y Canadá. Si Somos Americanos, v. 16, n. 2, p. 11-43, 2016.

66. THAYER, Luis E. Las paradojas de Moreno. Nuevo Poder, 24 de marzo de 2018. Disponible en: http://www.nuevopoder.cl/las-paradojas-de-moreno/

67. TOPAK, Özgün. The biopolitical border in practice: surveillance and death at the Greece-Turkey borderzones. Environment and Planning D: Society and Space, v. 32, n. 5, p. 815-833, 2014.

68. WAEVER, Ole. Securitization and desecuritization. En: LIPSCHUTZ, Ronnie (ed.). On security. Nueva York: Columbia University Press. 1998. p. 46- 87.

69. WALTERS, William. Border/control. European Journal of Social Theory, v. 9, n. 2, p. 187-203, 2006. 
